\title{
Gravitational astronomy 101
}

\author{
It's a bit of a shock.
}

\section{David Blair}

I want to start this course with a brief review of the history of gravitational astronomy, starting from the Eta Carinae supernova of 2009. You will be in mid-career when the shock front hits, and a good understanding of GA will be useful for anyone remotely involved in the Shield Project.

The UN declared 2009 to be an International Year of Astronomy, in honour of the 400th anniversary of Galileo's first observations with a telescope. Just 5 years before Galileo discovered craters on the Moon and Jupiter's moons, Kepler had recorded a supernova. From that date until 2009, for the entire time since the invention of the telescope, there had been a complete absence of supernovae in our Galaxy. Astronomers thought this very unfair. If you had been born in the year 1000 you could have seen two supernovae in your lifetime, and the same if you had been born in 1550 . But the unfairness of the 400 -year absence is nothing compared with the unfairness of the Eta Carinae supernova. For Earth, the coming shock is a one-in-a-million-year event.

But I digress. Let's focus on the gravitational astronomy. Over the years before the EC supernova, there had been many years of painstaking development of gravitational-wave detectors. The world detector array that now runs autonomously was in its infancy. There was not even one Southern Hemisphere detector. The angular resolution (which we will derive in the next lecture) was lousy.

The EC supernova was a major embarrassment to science. Even though electromag astronomers had been predicting it for half a century, no one noticed when it actually happened. The gravity signal was so different from any predicted waveform that it too was only identified months after the event. So the most momentous astronomical event in human history - one that, as you all know, threatens to put an end to human history - was ignored for days, until a few amateurs noticed the changes in Eta Carinae. The trouble was that Eta Carinae was very special and very rare: one star among 100 billion that was more than 100 times as heavy as our Sun. Its light was blanketed by the nebula and the prompt $\gamma$-ray burst lit up only a corner of the nebula.

Getting an accurate distance to EC was the key to predicting the shock arrival, which as you all know is expected in March '81. Like all gravity signals, the EC signal gave us its distance, which is 7,239 light years. You would think that was sufficiently far away for Earth to be safe. To see why it's not, you have to understand geons and strong-field nonlinear coupling in supersymmetric loop quantum gravity.

Geons were discovered theoretically by John Archibald Wheeler in 1955. They are gravitationally bound entities of pure gravitational energy. Most astrophysicists believe that they could not actually exist, despite Gell-Mann's totalitarian principle, which says if it is not prohibited then it is compulsory. Geons are not prohibited by the laws of physics so they must be out there. But Wheeler did not consider gravitinoneutralino coupling. This causes strongfield gravitational wavefronts to gravitationally condense into geon-like bubbles that propagate at about $0.99 c$. By the end of the course you should be able to derive the threshold gravitational-wave amplitude for the creation of gravitinoneutralino geons.

The year 2009 broke the supernova drought but it also gave us the great flood that is on its way. And for physicists it broke the drought in our understanding of the Universe. In 2009, physics had been derailed by an impossibly complex theory they called string theory. It was like Ptolemy's theory of the Solar System that derailed astronomy for more than a millennium. While physicists used string theory to try to solve an apparent incompatibility between quantum mechanics and gravity, astronomers were invoking stuff they called 'dark matter' and 'dark energy' to explain galaxy rotation and cosmic expansion. Physics was in a mess. The key to the great synthesis was in the 2009 gravity-wave data. The breakdown of general relativity at the black-hole event horizon seems obvious today, but it took Otulu 20 years to figure it out.

It is the nonlinearity of classical general relativity that causes the instabilities that turn shock fronts into cosmic bullets. This is the fundamental cause of the catastrophe of the coming shock and you must understand it in detail. At the end of the course I will expect you to be able to derive both the gravitinoneutralino shock-front structure as well as its peak intensity and arrival time from the gravity-wave data, using Otulu's strong-coupling theory. You will need to understand how the scalar field drives the coherent decay of the shock when it interacts with stellar plasma. You will need to be familiar with the extragalactic data that confirmed the theory, and be able to show how propagating gravitino-neutralino-geon condensates explain both cosmic-expansion data and galaxy rotation. This will test your understanding of the great synthesis.

The absence of other galactic civilizations is probably due to their failure to understand gravitino-neutralino geons. I hope that some of you will go on to work for the Shield Project and make sure we do not follow in their footsteps.

Now, let's get to work. The first slide shows the Otulu equation...

David Blair is director of the Australian International Gravitational Research Centre at the University of Western Australia, a co-founder of the Gravity Discovery Centre (www.gdc.asn.au) and Western Australia's Scientist of the Year. This story was inspired by discussions at CECS Chile.

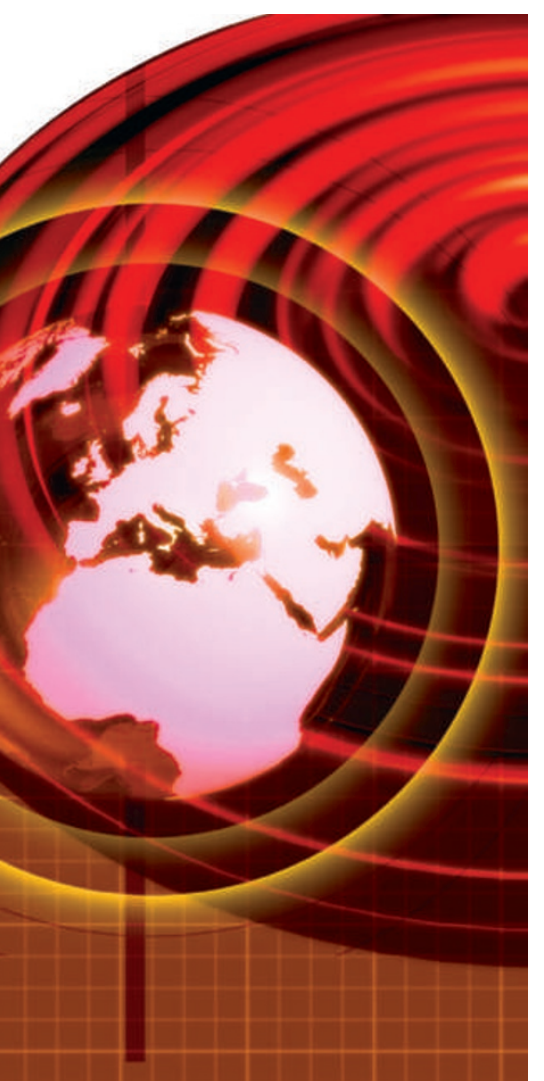

